\title{
COUPLED REAL-TIME THERMO-MECHANICAL SOLIDIFICATION MODEL OF CONTINUOUSLY CAST STEEL
}

\author{
Michal BŘEZINA, Tomáš MAUDER, Lubomír KLIMEŠ, Josef ŠTĚTINA, Tomáš NÁVRAT, \\ Jindřich PETRUŠKA
}

Brno University of Technology, Brno, Czech Republic, EU

Michal.Brezina1@vutbr.cz

https://doi.org/10.37904/metal.2019.791

\begin{abstract}
Current computational IT techniques allow to compute large complex multi-physical models and come up with a real-time solution. Due to this fact, a thermo-mechanical model for continuous steel casting can be created and used as a real-time crack prediction tool. Thermal and mechanical crack conditions must be evaluated to conclude whether the cast strand is of a sufficient quality suitable for its immediate next processing such as rolling. The previously developed solidification model BrDSM will be coupled with a newly developed mechanical stress-strain model. The crack criteria mentioned in this paper can be used as an optimization tool for the quality prediction of steel. Thus, this approach can be applied for the increase of the steel production due to the fast assessment of the strand quality based on the evaluation of critical crack conditions, which assure high quality steel. In the paper, the use of crack criteria as a quality predictor is outlined and the idea of the coupling between thermal and mechanical models is presented. The functionality of these models will be verified with data obtained from experimental measurements at a steel plant.
\end{abstract}

Keywords: Continuous casting, Crack criteria, Thermo-mechanical model, Quality prediction

\section{INTRODUCTION}

The method of continuous casting (CC) is a well-known process of converting molten steel to a solidified semifinished product: slab, billet, or bloom. The casting process is illustrated in Figure 1. From the tundish, molten steel is poured through the submerged nozzle into the primary cooling zone of the continuous casting machine (CCM), which is called the mold. In the mold the required shape of the semi-product is formed based on the shape of the water-cooled mold. Before the strand enters the next section called the secondary cooling zone, the strand needs to form a solidified shell on its surface to withstand the pressure from molten steel in the core of the strand. If the thickness of the shell is not sufficient, the breakdown can occur. To avoid such situations, a mathematical model calculating the temperature distribution of the strand must be deployed to set appropriate casting values, and thus to enable sufficient cooling in the mold, which forms the sufficient shell thickness. The strand moves from the mold to the secondary cooling zone where the strand is cooled mainly by air-mist nozzles, rollers, free convection and radiation to the ambient environment. This section withdraws a huge amount of heat and cools the strand such that the core is completely solidified as well. To be able to predict the location in which the strand (its cross-section) reaches the fully solidified state, a mathematical model is needed as well, because after that the strand is cut into pieces of a required length. These pieces are then moved to a place, where they are cooled down just due to radiation, and their quality is assessed afterwards. When the strand is cooled enough to be assessed, the decision is made. It can be either immediately further processed without any major interferences, or the surface needs to be scarfed to improve the quality of the slab. This part of the assessment takes a considerable amount of time, and also additional energy is needed to heat the strand again to an adequate temperature for subsequent rolling etc. It is reported in literature and industry data that about $16 \%$ of defects were due to faulty casting practice, $29 \%$ due to an inappropriate setup of casting facilities, $38 \%$ owing to incorrect process parameters, and $17 \%$ of defects were due to chemical composition of steel [1]. 


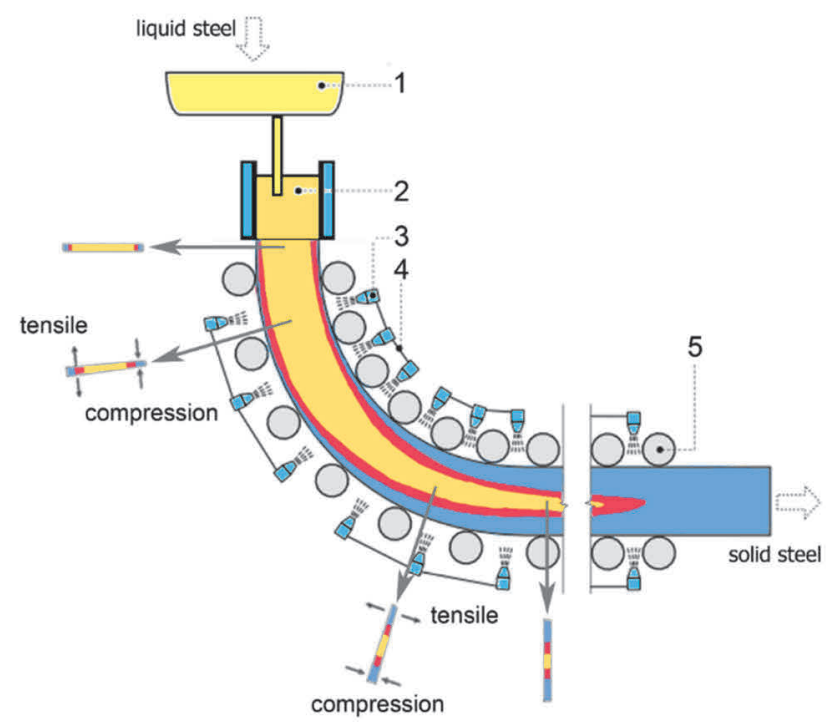

Figure 1 The CCM with depicted mechanical stresses during bending and straightening. 1 - tundish; 2 - mold; 3 - water-air nozzle; 4 - cooling circuit; 5 - roller [2]

Therefore, the power of the coupled real-time thermo-mechanical solidification model can take its place. It is known that the steel of a defined chemical composition is prone to cracking under certain circumstances. The idea is to utilize modern computational techniques, which can allow for the computation of complex multiphysical models on a very fine computational domain, and to use this advantage to assess if the product is or is not of a good quality without waiting until it cools down. To be able to predict the quality of the currently cast strand, the previously developed solidification model BrDSM must be re-implemented into the coupled thermo-mechanical model. The BrDSM model can predict the quality based on the temperature distribution only. But this approach is not sufficient and mechanical properties, which affect the strand, have to be considered as well. Once the thermo-mechanical model is created, it can be used to evaluate the so-called crack criteria. These criteria give information about the casting conditions, which affect the quality. Most cracks are transversal, longitudinal, and star cracks. The paper presented by Kulkarni and Babu [1] is the cornerstone of this work. They proposed 17 crack criteria, which give information about the quality of the strand. If the critical conditions are reached, it can be with a certain probability concluded whether the slab contains some defect or not. However, a problem is related with coefficients included in these criteria. Their model was designed for a specific CCM and these criteria are not applicable to any other CCM. The effort is to optimize these criteria to meet the quality casting of specific CCMs. In this paper the idea of this prediction/regulation system is outlined.

\section{THERMO-MECHANICAL MODEL}

The mathematical formulation of heat transfer and solidification to the temperature distribution and the solid shell profile prediction is based on the governing equation of transient heat conduction, often called the FourierKirchhoff equation [3]. In 3D the heat transfer equation reads

$\frac{\partial(\rho h)}{\partial t}+v \frac{\partial(\rho h)}{\partial z}=\nabla \cdot\left[k_{e f f}(T) \nabla T\right]$

where $k_{e f f}$ is the effective thermal conductivity $\left(\mathrm{W} \mathrm{m}^{-1} \mathrm{~K}^{-1}\right), \rho$ is the density $\left(\mathrm{kg} \mathrm{m}^{-3}\right) T$ is the temperature $(\mathrm{K}), h$ is the enthalpy $\left(\mathrm{J} \mathrm{kg}^{-1}\right), t$ is the time $(\mathrm{s}), v$ is the casting speed $\left(\mathrm{m} \mathrm{s}^{-1}\right)$ and $z$ is the direction of casting $(\mathrm{m})$.

The enthalpy can be expressed as follows [3]:

$h=\int_{0}^{T}\left(c_{p}-\Delta H \frac{\partial f_{s}}{\partial \xi}\right) d \xi$ 
where $\Delta H$ is the amount of latent heat $\left(\mathrm{J} \mathrm{kg}^{-1}\right), c_{p}$ is the specific heat capacity $\left(\mathrm{J} \mathrm{kg}^{-1} \mathrm{~K}^{-1}\right)$ and $f_{s}$ is the solid fraction (-). In order to define a well-posed problem, initial and boundary conditions must be provided. The boundary conditions include the initial strand temperature, heat fluxes to rollers, conditions for forced convection (cooling nozzles) and free convection, and radiation from surfaces of the strand.

While the strand is passing through the caster, it naturally varies its thermophysical properties. To express the relation between the enthalpy and the temperature, the thermophysical properties (density, specific heat, thermal conductivity, contraction, proof stress, shear modulus, etc.) are determined with the use of the solidification analysis package, called the IDS [4] (interdendritic solidification software), which is reliable and practical for the use rather than expensive and time-consuming experimental investigations.

From the mechanical point of view, two different types of the stress are mainly involved in CC: thermal stress and mechanical stress. The thermal stress in the strand is generated by a non-homogenous temperature distribution. Sources of the mechanical stress are mainly the friction in the mold, ferrostatic forces due to the liquid steel core, forces due to rollers, and bending and straightening mills. There are several sources, which give rise to the evolution of mechanical stress in the solidifying strand. These include volumetric changes due to temperature gradients and phase transformations, mechanical loading of the shell from the ferrostatic pressure of the liquid steel, and external loading from support rollers and from the global deformation [5]. In order to describe the complete temperature range of solidification and cooling, a generalized elastoviscoplastic constitutive model [6]

$\dot{\varepsilon}=\dot{\varepsilon}^{e l}+\dot{\varepsilon}^{v p}+\dot{\varepsilon}^{t h}=\frac{1+v}{E} \dot{\boldsymbol{\sigma}}-\frac{v}{E} \operatorname{tr}(\dot{\boldsymbol{\sigma}}) \boldsymbol{I}+\frac{3}{2 \sigma_{e q}}\left[\frac{\sigma_{e q}-\sigma_{0}}{K \varepsilon_{e q}^{n}}\right]^{\frac{1}{m}} \boldsymbol{s}+\alpha \dot{T} \boldsymbol{I}$

can be adopted to describe the evolution of stress, strain and strain rate fields. The efficiency of the computational analysis can be improved by additional assumptions concerning the geometry of the solidified body as in case of the axi-symmetry or in case of the generalized plane strain, which reduces six independent components of each tensor in equation (3) to four or even three components [7]. The strain rate tensor $\dot{\varepsilon}$ is split into the elastic $\dot{\varepsilon}^{e l}$, viscoplastic $\dot{\varepsilon}^{v p}$, and thermal part $\dot{\varepsilon}^{t h}$. The elastic component in equation (3) is described by the Hooke's law and is related to temperature-dependent material parameters: the Young's modulus $E$ and the Poisson coefficient $v$. The equivalent stress in the viscoplastic part of equation (3) can be expressed as [8]

$\sigma_{e q}=\sigma_{0}+K \varepsilon_{e q}^{n} \varepsilon_{e q}^{m}$

where $\sigma_{0}$ denotes the static yield stress below which no viscoplastic deformation occurs. The last term in equation (3) describes the thermal strain caused both by temperature-induced volume changes and phase transformations, both related to a temperature-dependent thermal expansion coefficient $\alpha$. In case of liquid and mushy states, an effective lowering of the yield stress to almost zero and an appropriate change in the material consistency leads to an extensive shear deformation according to the Newtonian fluid dynamics. Nevertheless, some non-zero yield stress is still kept to avoid numerical difficulty [7]. Other variables in equation (3) are: $\dot{T}$ denotes the temperature rate, $K$ is the so-called consistency of the material, $m$ is the strain rate sensitivity coefficient, $I$ is the identity tensor, and $s$ is the stress deviator defined in [8].

A solution of the above given equations represents a complex problem, especially due to the coupling of the thermo-mechanical phenomena and due to the lack of reliable temperature-dependent material parameters. The analyzed history of stress and strain distributions during the solidification is the basis for the prediction of both the high temperature fracture risk and residual stresses at the end of the process. In order to validate the above described models, the stress state in the solidified strand can be measured by the RESTAN approach, see e.g..

Two-dimensional models are in these days replaced by three-dimensional (3D) models. However, a weak point of these thermal-mechanical models is the computation time (cost), which is much higher than the wall-clock 
time of the process being simulated. Real-time simulations or even the real-time control of the CC process is therefore beyond the limit and capabilities of such models. The goal is to create a coupled thermal-mechanical model of the solidifying strand including both the liquid and solid phases. The model will be fully 3D, transient, and implemented in a form suitable for the GPU (Graphics Processing Unit) architecture. The GPU approach allows for massive parallel processing and real-time simulations [10]. The developed thermal-mechanical model validated with the use of experimentally gained data can be then used to establish, evaluate, and test thermal-mechanical criteria, which serve for the prediction of crack and defect formation from the distribution of temperature and stresses.

\section{CRACK CRITERIA}

The crack formation has plagued the CC method since its inception, and quality issues limit the range of steel grades that a particular caster can cast and produce. Knowledge of the temperature distribution in the strand together with the mechanical strain and stress distribution is required to evaluate the risk of initiation of surface cracks. Usually used criteria and indicators for the crack origin in the solid state are based on local thermos-mechanical variables [5]. The most frequent surface defects are transversal cracks, which are particularly caused by deep oscillation marks followed by the tension generated by bulging, bending, and straightening of the strand. There is also an influence of the thermal stress, which results from an uneven cooling of the strand shell in the secondary cooling zone [1]. Moreover, the origin of cracks caused by bending and straightening processes is influenced by the ductility of steel as well.

In order to obtain a defect free strand, five major properties should be taken into consideration. These include the prediction of the solidus temperature, the zero ductility temperature (ZDT), the liquid impenetrable temperature (LIT), the allowable thermal strain, the critical stress, and the casting speed as the function of the shell thickness at the mold exit. Steel grades with a larger difference between LIT and ZDT have a higher tendency for hot cracking, and thus they have a higher tendency towards defects, such as internal cracking. It is therefore useful to estimate ZDT and LIT, and to predict the crack formation during the casting process. ZDT and LIT mainly depend on the chemical composition. Many models for the crack prediction require stress and strain distributions in the material subjected to mechanical and thermal loads. The further step of the prediction procedure is the selection of a suitable crack criteria, which allows for the identification where and when the crack can occur. However, the selection of a suitable criteria is a difficult task, in which data available for the analysis must be considered.

A number of criteria related to various quality parameters of steel exist. Some criteria are discussed in [1] and those were proposed and used for optimization of free defect casting. One of the criteria is the determination of the maximum allowable stress in the strand shell at the mold exit, preventing the liquid steel in the core to penetrate out in case the thickness of the solid shell is not sufficient

$\sigma_{T}=C_{1}+C_{2}($ wide face dimension $)+C_{3}($ mold length $)+C_{4}($ roll pitch $)+C_{5}($ caster radius $)+$

$C_{6}($ shell thickness $)+C_{7}($ LIT $)+C_{8}($ thermal strain $)+C_{9}($ surface temperature at mold exit $)<0.95 \sigma_{c}$

where $\sigma_{T}$ is the total stress in the strand shell, $\sigma_{c}$ is the critical stress for cracking being a function of the chemical composition, and $C_{1}$ to $C_{9}$ are constants specified in [1]. Another condition, which considers the maximum allowable reheating $\Delta t_{\max }$ of the strand shell behind the spray zone, is evaluated to avoid stresses due to bulging as

$\Delta t_{\text {max }}=K_{1}+K_{2}$ (spray exit shell thickness $)+K_{3}$ (solidus temperature of steel) + $K_{4}$ (spray exit temperature) $<80^{\circ} \mathrm{C}$

where $K_{1}$ and $K_{4}$ are coefficients. 


\section{OPTIMIZATION OF CONTINUOUS CASTING PROCESS USING CRACK CRITERIA}

The identification of free defect casting based on the evaluation of crack criteria is a difficult task. First, the use of thermo-mechanical model and data from a steel plant about the quality are needed to identify suitable crack criteria. The idea of the approach is depicted in Figure 2. Casting parameters can be set up based on the quality reports and used in the calculation of thermal and mechanical distributions. Calculated values are then substituted into the criteria and compared with the quality reports. The criteria optimization consists in obtaining the same quality results in both quality reports and prediction results based on the values of criteria obtained by the model.

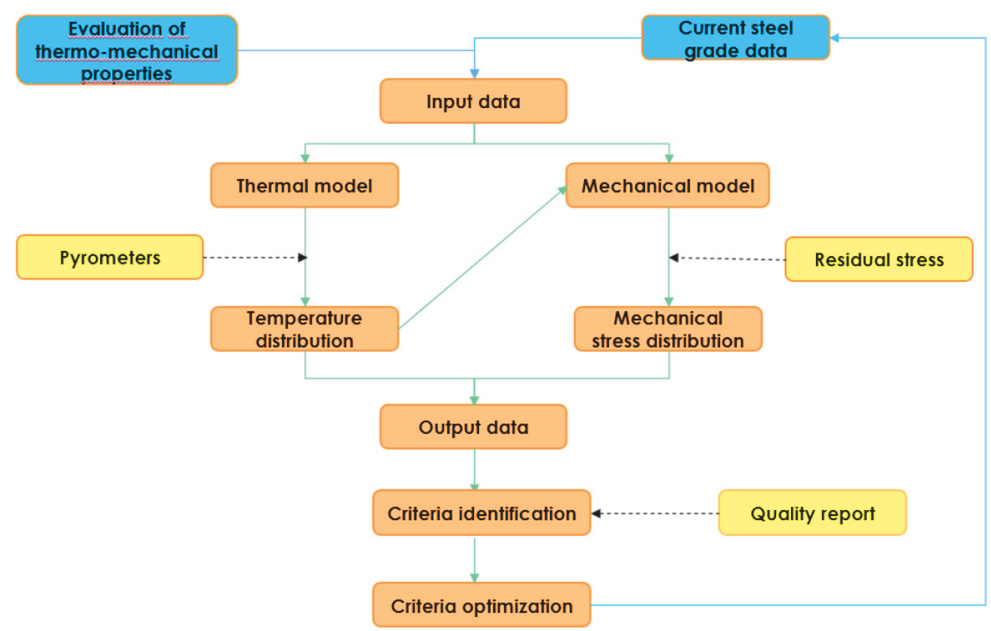

Figure 2 Identification of crack criteria

The idea of the regulation/prediction system is shown in Figure 3. The rapid simulation of thermal and mechanical models can be achieved by means of GPU techniques, which provide the fast evaluation of temperature and stress-strain distributions with a subsequent assessment of the strand quality based on the crack criteria. If none of the critical condition is violated, the strand can be considered as a strand without quality issues and can be immediately further processed.

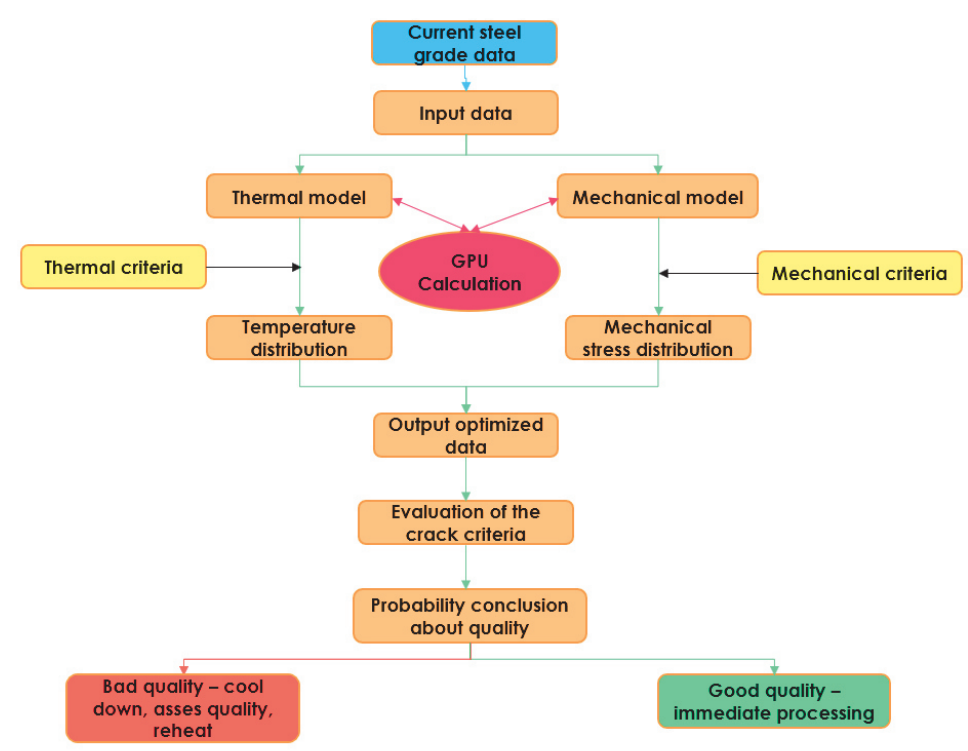

Figure 3 Optimization of the CC process with the use of crack criteria, with a subsequent assessment of the strand quality 


\section{CONCLUSION}

Though the continuous casting method is a matured technology for the steel production, there is still a considerable effort for the solution of quality issues, for the prediction of defects, and for the minimization of their occurrence. The concept of the coupled thermal-mechanical model for the prediction of the crack formation in continuous steel casting is presented in the paper. The coupled model is based on the use of two main sub-models. The thermal model, which allows for the determination of the temperature distribution and solidification, is utilized for the evaluation of the thermal stress. The other model is devoted for the solution of mechanical phenomena and stress-strain distribution in the strand. Their coupling into the thermal-mechanical model enables the evaluation of the quality criteria adopted from literature, which can be used for the identification of the crack origin and crack formation. Since the solution of the thermal as well as mechanical models is computationally expensive, an effective implementation of the coupled model is proposed for its solution by means of graphics processing units (GPUs). Achieved results indicate that the GPU approach can significantly speed up the simulation procedure, which makes it applicable in the real-time use at steel plants. As for the further steps, experimental investigations will be carried out at a steel plant, and data acquired experimentally will serve for the assessment, validation, and improvement of the proposed model.

\section{ACKNOWLEDGEMENTS}

This work was supported by the Czech Science Foundation under contract No. 19-20802S "A coupled real-time thermo-mechanical solidification model of steel for crack prediction" and by the project of Brno University of Technology, No. FSI-S-17-4444

\section{REFERENCES}

[1] KULKARNI, M.S., BABU, A. S. Managing quality in continuous casting process using product quality model and simulated annealing. J. Mater. Process. Technol., 2005, vol. 166, no. 12, pp. 294-306.

[2] MAUDER, T., STETINA, J. High quality steel casting by using advanced mathematical methods. J. METALS, 2018.vol. 8, no. 12, doi: 10.3390/met8121019.

[3] STEFANESCU, D. M. Science and Engineering of Casting Solidification. Second Edition, New York, Springer Science, (2009), 402 p., ISBN 978-0-387-74609-8.

[4] MIETTINEN, J., LOUHENKILPI, S., KYTÖNEN, H. and LAINE, J,. IDS: Thermodynamic-kinetic-empirical tool for modelling of solidification, microstructure and material properties. Mathematics and Computers in Simulation [online]., 2010, 80(7), 1536-1550. ISSN 03784754. Avaiable from: doi:10.1016/j.matcom.2009.11.002.

[5] BELLET, M., HEINRICH, A. A Two-dimensional Finite Element Thermomechanical Approach to a Global StressStrain Analysis of Steel Continuous Casting. ISIJ Int., 2004, vol. 44, no. 10, pp. 1686-1695.

[6] KORIC, S., HIBBELER, L. C., LIU, R., THOMAS, B. G. Multiphysics model of metal solidification on the continuum level, Numer. Heat Transf. Part B Fundam., 2010, vol. 58, no. 6, pp. 371-392.

[7] LI, C., THOMAS, B. G. Thermomechanical finite-element model of shell behavior in continuous casting of steel," Metall. Mater. Trans. B, 2004, vol. 35, no. 6, pp. 1151-1172.

[8] KELLY, J. E., MICHALEK, K. R., O'CONNOR, T. G., THOMAS, B. G. Initial Development of Thermal and Stress Fields in Continuously Cast Steel Billets, Metall. Trans., 1988 A, vol. 19, no. 10, pp. 2589-2602.

[9] SANTANA, Y. Y., et al. Measurement of residual stress in thermal spray coatings by the incremental hole drilling method, Surf. Coatings Technol., 2006, vol. 201, no. 5, pp. 2092-2098.

[10] KLIMES, L., STETINA, J., Parallel dynamic solidification model of continuous steel casting on GPU. In METAL 2013: $22^{\text {nd }}$ International Conference on Metallurgy and Materials. Brno: TANGER, 2013, pp. 74-79. 